OPEN ACCESS

Edited by:

John W. A. Rossen,

University Medical Center Groningen,

Netherlands

Reviewed by:

Alejandra Krüger,

National University of Central Buenos

Aires, Argentina

Eelco Franz,

Centre for Infectious Disease Control,

Netherlands

*Correspondence:

Yanwen Xiong

xiongyanwen@icdc.cn;

Shan Lu

lushan@icdc.cn

Specialty section: This article was submitted to

Infectious Diseases,

a section of the journal

Frontiers in Microbiology

Received: 16 February 2016 Accepted: 08 March 2016

Published: 22 March 2016

Citation:

Bai $X$, Zhang W, Tang $X, X$ in $Y, X u Y$,

Sun $H, L$ Lo $X, P u J$ J $X u$ J, Xiong $Y$

and Lu S (2016) Shiga

Toxin-Producing Escherichia coli

in Plateau Pika (Ochotona curzoniae)

on the Qinghai-Tibetan Plateau,

China. Front. Microbiol. 7:375

doi: 10.3389/fmicb.2016.00375

\section{Shiga Toxin-Producing Escherichia coli in Plateau Pika (Ochotona curzoniae) on the Qinghai-Tibetan Plateau, China}

\author{
Xiangning Bai ${ }^{1}$, Wang Zhang ${ }^{1}$, Xinyuan Tang ${ }^{2}$, Youquan Xin ${ }^{2}$, Yanmei Xu' ${ }^{1}$, Hui Sun', \\ Xuelian $\mathrm{LuO}^{1}$, Ji Pu' ${ }^{1}$, Jianguo $\mathrm{Xu}^{1,3}$, Yanwen Xiong ${ }^{1,3 *}$ and Shan $\mathrm{Lu}^{1 *}$
}

'State Key Laboratory for Infectious Disease Prevention and Control, National Institute for Communicable Disease Control and Prevention, Chinese Center for Disease Control and Prevention, Beijing, China, ${ }^{2}$ Qinghai Institute for Endemic Disease Prevention and Control, Xining, China, ${ }^{3}$ Collaborative Innovation Center for Diagnosis and Treatment of Infectious Diseases, Hangzhou, China

Shiga toxin-producing Escherichia coli (STEC) are an emerging group of zoonotic pathogens. Ruminants are the natural reservoir of STEC. In this study we determined the prevalence and characteristics of the STEC in plateau pika (Ochotona curzoniae) on the Qinghai-Tibetan Plateau, China. A total of 1116 pika samples, including 294 intestinal contents samples, 317 fecal samples, and 505 intestinal contents samples, were collected from May to August in the years 2012, 2013, and 2015, respectively. Twenty-one samples (1.88\%) yielded at least one STEC isolate; in total, 22 STEC isolates were recovered. Thirteen different $O$ serogroups and 14 serotypes were identified. One $s t x_{1}$ subtype $\left(s t x_{1 a}\right)$ and three $s t x_{2}$ subtypes ( $s t x_{2 a}, s t x_{2 b}$, and $\left.s t x_{2 d}\right)$ were present in the STEC isolates. Fifteen, fourteen, and three STEC isolates harbored the virulence genes ehxA, subA, and ast $A$, respectively. Adherence-associated genes iha and saa were, respectively, present in 72.73 and $68.18 \%$ of the STEC isolates. Twenty antibiotics were active against all the STEC isolates; all strains were resistant to penicillin $\mathrm{G}$, and some to cephalothin or streptomycin. The 22 STEC isolates were divided into 16 pulsed-field gel electrophoresis patterns and 12 sequence types. Plateau pikas may play a role in the ongoing circulation of STEC in the Qinghai-Tibetan plateau. This study provides the first report on STEC in plateau pikas and new information about STEC reservoirs in wildlife. Based on the serotypes, virulence gene profiles and multi-locus sequence typing (MLST) analysis, the majority of these pika STECs may pose a low public health risk.

Keywords: Shiga toxin-producing Escherichia coli, plateau pika, MLST, PFGE, antibiotic resistance

\section{INTRODUCTION}

Shiga toxin-producing Escherichia coli (STEC) represents an emerging group of zoonotic pathogens causing diarrhea, hemorrhagic colitis (HC), and the life-threatening hemolytic uremic syndrome (HUS) in humans (Smith et al., 2014). Strains of O157 serogroup have been considered to be most virulent, and STEC O157 strains have been extensively studied and shown to be involved in many cases and outbreaks of human disease (Doorduyn et al., 2006; Friesema et al., 2007; 
Sigmundsdottir et al., 2007; Greenland et al., 2009). However, there is growing concern over the emergence of more than 200 non-O157 STEC serotypes associated with human illness, including the life threatening HUS (Coombes et al., 2008). In fact, non-O157 strains are responsible for a larger portion of STEC infections than O157 strains in the United States, Canada, Australia, Latin America, and Europe (Karmali et al., 1985; Caprioli et al., 1997; Tozzi et al., 2003; Brooks et al., 2005). At present, detection methods and in depth studies are mainly focused on O157:H7 and the top six serogroups of STEC (O26, O45, O103, O111, O121, and O145) (Conrad et al., 2014; Wasilenko et al., 2014), thus the public health significance of STEC of other serotypes is likely to be underestimated due to the high diversity of genotypes and phenotypes.

Shiga toxin (Stx) is the most critical virulence factor of STEC, consisting of two broad immunologically different types: Stx1 with three subtypes (Stxla, Stx1c, and Stxld) and Stx2 with seven subtypes (Stx2a to Stx2g). Stx can damage intestinal epithelial cells and kidneys, causing $\mathrm{HC}$ and HUS (Smith et al., 2014). However, many non-O157 strains that produce Shiga toxin have been not associated with HUS cases, indicating additional virulence determinants may play a role in pathogenesis. Following initial attachment of STEC to host intestinal cells, strains that express intimin can intimately attach to host cells and cause the attaching-and-effacing lesion (Frankel and Phillips, 2008). Intimin is encoded by eae that resides on a chromosomal pathogenicity island called the locus of enterocyte effacement (LEE). Enterohemolysin (EhxA), encoded by a $60-\mathrm{MDa}$ virulence plasmid in some STEC strains, that readily causes hemolysis of washed sheep erythrocytes, is another important virulence factor that contributes to severe disease in humans (Cookson et al., 2007). A number of other adherence structures and virulence factors have also been proposed to contribute to STEC pathogenesis (Coombes et al., 2008; Tseng et al., 2014; Chui et al., 2015).

Though the main reservoir of STEC is the intestinal tract of cattle, many other domestic and wild animals including pig, sheep, dog, deer, wild boar, and hare are also sources of STEC (Oporto et al., 2008; Miko et al., 2009; Sanchez et al., 2010; Bentancor et al., 2012; Shen et al., 2015). STEC strains have been described recently in diarrheal patients, domestic animals and foodstuffs of animal origin in China (Xiong et al., 2012; Chen et al., 2014; Meng et al., 2014; Bai et al., 2015; Wang et al., 2015; Yu et al., 2015), but little data is available concerning wildlife. The plateau pikas (Ochotona curzoniae) are underground-dwelling mammals (order Lagomorpha) mostly living in the extremely harsh wild environments of severe cold, low atmospheric oxygen and strong ultraviolet radiation of the Qinghai-Tibetan plateau, China. Plateau pikas have close contact with yaks. In our previous study, we explored the occurrence and characteristics of STEC in yaks (Bos grunniens) for the first time, revealing that yaks are natural reservoirs of STEC, and some of the STEC strains they harbored had the potential to cause human disease (Bai et al., 2013). In this study, we investigated the prevalence of STEC in plateau pikas in this unique ecosystem.

\section{MATERIALS AND METHODS}

\section{Collection of Samples}

Four sites were enrolled in this study in Yushu tibetan autonomous prefecture, Qinghai province, China, including Guoqinggou [3,929 $\mathrm{m}$ above msl (mean sea level), latitude of $33^{\circ} 7^{\prime}$ and longitude of $\left.96^{\circ} 84^{\prime}\right)$, Jielachong (3,970 $\mathrm{m}$ above msl, latitude of $33^{\circ} 48^{\prime}$ and longitude of $\left.96^{\circ} 51^{\prime}\right)$, Gandacun $(4,322 \mathrm{~m}$ above msl, latitude of $33^{\circ} 13^{\prime}$ and longitude of $96^{\circ} 73^{\prime}$ ), Batangtan $\left(3,987 \mathrm{~m}\right.$ above $\mathrm{msl}$, latitude of $32^{\circ} 51^{\prime}$ and longitude of $\left.96^{\circ} 56^{\prime}\right)$ ]. The plateau pikas were captured by 'mousetrap' in their breeding seasons from May to August when their population densities are high, and the colon contents were collected. Fresh feces were picked up from the holes. In total, 1116 samples were collected consisting of 294 colon contents samples, 317 fecal samples, and 505 colon contents samples in the years 2012, 2013, and 2015, respectively. Samples were mixed in Luria-Bertani medium containing 30\% glycerol (Land Bridge, Beijing, China), stored at $-20^{\circ} \mathrm{C}$ immediately, and transported to the laboratory in National Institute for Communicable Disease Control and Prevention, China CDC, in ice cold conditions for the screening of STEC.

\section{Isolation of STEC}

One gram of each sample was enriched into $5 \mathrm{ml}$ of $E$. coli broth (Land Bridge, Beijing, China) and incubated at $37^{\circ} \mathrm{C}$ for 18 to $24 \mathrm{~h}$ with shaking at $200 \mathrm{rpm}$. The enriched samples were investigated for $s t x_{1}$ and $s t x_{2}$ genes by duplex PCR assay as used in our previous study (Bai et al., 2015). Briefly, $1.5 \mathrm{ml}$ of each enrichment sample was centrifuged at $13,000 \times g$ for $2 \mathrm{~min}$, the pellet was suspended in $150 \mu \mathrm{l}$ of lysis buffer $(100 \mathrm{mM} \mathrm{NaCl}$, $10 \mathrm{mM}$ Tris- $\mathrm{HCl}$ [pH 8.3], 1 mM EDTA [pH 9.0], 1\% Triton $\mathrm{X}-100$ ), then boiled for $10 \mathrm{~min}$, and centrifuged at $13,000 \times g$ for $2 \mathrm{~min}$. The supernatant was then used as the PCR template. Enriched samples that tested positive for $s t x_{1}$ and/or $s t x_{2}$ genes were plated onto CHROMagar ${ }^{\mathrm{TM}}$ ECC agar (CHROMagar, Paris, France), and MacConkey agar (Oxoid, Hampshire, UK) and then incubated at $37^{\circ} \mathrm{C}$ overnight. About 10 colonies (blue or colorless, round moist E. coli-like colonies) on CHROMagar ${ }^{\text {TM }}$ ECC and 5 pink or red colonies on MacConkey were picked to screened for the presence of $s t x_{1}$ and/or st $x_{2}$ genes by single colony duplex PCR assay (Bai et al., 2015). If all colonies were negative for stx, another 10 and 5 colonies on the two plates were picked and screened. Finally, with the exception of different colony colors (blue or colorless), stx types or stx combinations present in the same sample, only one STEC isolate from each stx-positive sample was kept for further identification as described previously (Bai et al., 2015).

\section{Biochemical Tests and Serotyping of STEC Isolates}

Each stx-positive isolate was confirmed to be $E$. coli by biochemical identification using the API 20E system (bioMérieux, Marcy l'Etoile, France). The O serogroup was determined using all available E. coli antisera, i.e., O1-O188 (Statens Serum Institut, Hillerød, Denmark). The entire coding 
sequence of $f l i C$ was amplified by PCR using primers F-FLIC1 (5'-ATGGCACAAGTCATTAATACCCAAC- $\left.3^{\prime}\right)$ and R-FLIC2 ( $5^{\prime}$-CTAACCCTGCAGCAGAGACA- $\left.3^{\prime}\right)$, as reported by Fields et al. (1997), then sequenced and compared to a publicly available CGE SerotypeFinder web tool ${ }^{1}$ to determine the $\mathrm{H}$ type of each isolate (Joensen et al., 2015).

\section{stx Subtyping}

The $s t x_{1}$ subtypes of STEC isolates were determined by a PCRbased subtyping method devised by Scheutz et al. (2012). The complete $s_{x_{2}}$ gene was amplified as described by Gunzer et al. (1992), then cloned into vector pMD18-T and transformed into E. coli JM109 (Takara, Dalian, China). About 10 transformants were selected for sequencing to discern multiple stx $x_{2}$ subtypes in a PCR product. A neighbor-joining tree was constructed using MEGA 6 (Tamura et al., 2013) to assign the $s t x_{2}$ subtype (Scheutz et al., 2012).

All stx 2 nucleotide sequences determined in this study have been submitted to GenBank under accession numbers KU158844-KU158861.

\section{Detection of Virulence and Adherence Factors}

Shiga toxin-producing Escherichia coli isolates were subjected to PCR for detection of intimin-encoding gene (eae), putative adhesin genes (iha, efa1, saa, paa, eibG), virulence-associated genes (ehxA, katP, toxB, ast $A, \operatorname{sub} A$ ), the high-pathogenicity island (HPI) marker genes (irp2 and $f y u A$ ) and 11 non-LEE encoded effector (nle) genes (ent, nleA, nleB, nleB2, nleE, nleH1-1, nleH1-2, nleC, nleD, nleF, nle G) using primers listed in Supplementary Table S1.

\section{Antimicrobial Susceptibility Testing}

Antimicrobial drug susceptibility was determined by the disk diffusion method as recommended by the Clinical and Laboratory Standards Institute (Clinical Laboratory Standards Institute [CLSI], 2014). We tested the following 23 antimicrobial agents: penicillin G, amoxicillin/clavulanic acid, ampicillin/ sulbactam, levofloxacin, nitrofurantoin, aztreonam, norfloxacin, chloramphenicol, cefepime, cephalothin, meropenem, ceftriaxone, imipenem, streptomycin, ciprofloxacin, gentamicin, piperacillin, cefotaxime, nalidixic acid, kanamycin, trimethoprim-sulfamethoxazole, cefuroxime, and tetracycline (Oxoid, Hampshire, UK). Results were used to classify isolates as being resistant or susceptible to a particular antibiotic using standard reference values (Clinical Laboratory Standards Institute [CLSI], 2014).

\section{Pulsed-Field Gel Electrophoresis (PFGE)}

Shiga toxin-producing Escherichia coli isolates were digested with $\mathrm{XbaI}$ and separated by pulsed-field gel electrophoresis (PFGE) according to the protocol for non-O157 STEC from PulseNet, $\mathrm{USA}^{2}$. Gel images were captured with a Gel Doc ${ }^{\mathrm{TM}} \mathrm{XR}+$

${ }^{1}$ http://cge.cbs.dtu.dk/services/

${ }^{2}$ http://www.cdc.gov/pulsenet/pathogens/index.html system (Bio-Rad, Hercules, CA, USA). An UPGMA (unweighted pair-group method with arithmetic mean) dendrogram was constructed using BioNumerics software version 4.0 (Applied Maths, Sint-Martens-Latem, Belgium).

\section{Multi-locus Sequence Typing (MLST)}

All STEC isolates were analyzed by multi-locus sequence typing (MLST) according to the E. coli MLST website 3 . Sequences types (STs) of the HUSEC (HUS-associated enterohemorrhagic E. coli) collection were obtained from http://campus.uni-muenster. de/hyg_klhus_husec.html? \&L $=1$ (Mellmann et al., 2008). All human STEC STs of O157 and the top six serogroups were obtained from the E. coli MLST website. A minimum spanning tree based on these STs was generated using BioNumerics software.

\section{Statistical Analysis}

The $\chi^{2}$ test was performed using SAS software version 9.1 (SAS Institute Inc., Cary, NC, USA). $P<0.05$ was considered statistically significant.

\section{Ethics Statement}

The pikas in this study were captured by 'mousetrap' by local center for disease control and prevention for communicable diseases surveillance. The study was approved by the ethics committee of National Institute for Communicable Disease Control and Prevention, China CDC, according to the medical research regulations of National Health and Family Planning Commission of the People's Republic of China.

\section{RESULTS}

\section{Prevalence of STEC in Plateau Pika Samples}

Twenty-two STEC isolates were obtained from 21 of the 58 stx-positive plateau pika samples giving a culture positive rate of $36.21 \%$ for stx-positive samples and $1.88 \%$ for all samples (Table 1). Single isolate was obtained from 19 intestinal content samples and one fecal sample. Two isolates (15ST443 and 15ST444) with different stx types were recovered from one intestinal contents sample collected in 2015. Nine out of 294 intestinal contents samples collected in 2012, one out of $317 \mathrm{fecal}$ samples collected in 2013, and 11 out of 505 intestinal contents samples yielded STEC isolates, giving culture positive rates of $3.06,0.32$, and $2.18 \%$ for all samples, respectively (Table 1). The culture positive rates in intestinal contents were much higher than that in feces $(P<0.05)$.

\section{Serogroups and Serotypes}

In total, 13 different $\mathrm{O}$ serogroups and 7 different $\mathrm{H}$ types were identified among the 22 STEC isolates, which belonged to 14 serotypes: O2:H45, O8:H2, O8:H16, O49:H21, O74:H8, O81:H21, O82:H19, O96:H8, O119:H19, O120:H9, O159:H21,

${ }^{3} \mathrm{http} / / / \mathrm{mlst}$. warwick.ac.uk/mlst/dbs/Ecoli 
O163:H19, O169:H8, and O170:H8. The predominant serotypes were $\mathrm{O} 8: \mathrm{H} 2, \mathrm{O} 2: \mathrm{H} 45, \mathrm{O} 74: \mathrm{H} 8$, and $\mathrm{O} 49: \mathrm{H} 21$ of which we found four $(18.18 \%)$, three $(13.64 \%)$, three $(13.64 \%)$ and two $(9.09 \%)$ isolates, respectively. The remaining 10 serotypes were each represented by only one isolate (Figure 1).

\section{Presence of stx Genes and stx Subtypes and Other Virulence Factor Genes}

Among the 22 STEC isolates, five tested positive for st $x_{1}$ only, 14 for $s t x_{2}$ only, and three for both $s t x_{1}$ and $s t x_{2}$. All of the eight $s t x_{1}$ were subtyped into $s t x_{1 a}$. Of the 17 st $x_{2}$-positive STEC isolates, one was subtyped into $s t x_{2 a}$, five were $s t x_{2 b}$,
10 were $s t x_{2 \mathrm{~d}}$, and one isolate carried both $s t x_{2 a}$ and $s t x_{2 \mathrm{~d}}$ (Figure 1).

All 22 STEC isolates were eae negative. Of the five other putative adhesin genes (iha, saa, efa1, paa, eibG) screened, iha and saa were present in $16(72.73 \%)$ and $15(68.18 \%)$ isolates, respectively, and the 15 saa positive STEC isolates also carried iha. The other three adhesin genes were not detected in any of the 22 isolates. We also screened five virulenceassociated genes (ehxA, katP, toxB, ast $A, \operatorname{sub} A)$, two markers for HPI (irp2 and $f y u A$ ), and 11 non-LEE encoded effector (nle) genes. Fifteen (68.18\%) STEC isolates were positive for ehxA, fourteen $(63.64 \%)$ and three (13.64\%) were positive for $s u b A$ and $a s t A$, respectively. All our STEC isolates were

TABLE 1 | Prevalence of Shiga toxin-producing Escherichia coli in plateau pika.

\begin{tabular}{|c|c|c|c|c|c|}
\hline Year & Type of sample & $\begin{array}{l}\text { Number of } \\
\text { samples }\end{array}$ & $\begin{array}{c}\text { Number of stx } \\
\text { positive (\%) }\end{array}$ & $\begin{array}{c}\text { Number of samples with } \\
\text { STEC isolates (\%) }\end{array}$ & $\begin{array}{c}\text { Number of STEC } \\
\text { isolates (\%) }\end{array}$ \\
\hline 2012 & Intestine contents & 294 & $10(3.40)$ & $9(3.06)$ & $9(3.06)$ \\
\hline 2013 & Feces & 317 & $2(0.63)$ & $1(0.32)$ & $1(0.32)$ \\
\hline 2015 & Intestine contents & 505 & $46(9.11)$ & $11(2.18)$ & $12(2.38)$ \\
\hline Total & & 1116 & $58(5.20)$ & $21(1.88)$ & $22(1.99)$ \\
\hline
\end{tabular}

\begin{tabular}{|c|c|c|c|c|c|c|c|c|c|c|c|c|c|c|}
\hline Similarity (\%) & non-0157 STEC & Isolate & PFGE pattern & ST & Serotype & stx & $s t x_{2} e$ & $e h x A$ & iha & sac & subA & asta & $\mathbf{S}$ & $\mathbf{K F}$ \\
\hline & & $15 S T 445$ & EMSX11001 & ST737 & O81:H21 & - & $2 a$ & + & + & + & + & - & - & - \\
\hline & & 15ST449 & EMSX11002 & ST3884 & O163:H19 & - & $2 \mathbf{b}$ & - & - & - & - & - & - & - \\
\hline & & 15ST441 & EMSX11003 & ST40 & O49:H21 & - & $2 \mathbf{b}$ & - & - & - & - & - & + & - \\
\hline & & 15ST444 & EMSX11003 & ST40 & O49:H21 & - & $2 b$ & - & - & - & - & - & - & - \\
\hline & & $15 S T 439$ & EMSX11004 & ST297 & O169:H8 & - & $2 b$ & + & + & + & + & - & - & - \\
\hline & & $15 S T 446$ & EMSX11005 & ST297 & O96:H8 & - & 2d & + & + & + & + & - & - & - \\
\hline & & $12 \mathrm{ST} 457$ & EMSX11006 & ST297 & O170:H8 & - & $2 d$ & + & + & + & + & - & - & - \\
\hline & & $15 S T 448$ & EMSX11007 & ST5653 & O120:H9 & 1a & 2d & + & + & + & - & - & - & + \\
\hline & & $15 S T 450$ & EMSX11008 & ST5741 & 0159:H21 & $1 \mathrm{a}$ & 2d & + & + & + & - & - & - & - \\
\hline & & $12 \mathrm{ST} 452$ & EMSX11009 & ST1001 & O8:H2 & - & 2d & + & + & + & + & - & - & - \\
\hline & & $12 \mathrm{ST} 453$ & EMSX11009 & ST1001 & O8:H2 & - & 2d & + & + & + & + & - & - & - \\
\hline & & $12 \mathrm{ST454}$ & EMSX11009 & ST1001 & O8:H2 & - & 2d & + & + & + & + & - & - & - \\
\hline & & $12 \mathrm{ST} 459$ & EMSX11009 & ST1001 & O8:H2 & - & 2d & + & + & + & + & - & - & - \\
\hline & & $15 \mathrm{ST} 443$ & EMSX11010 & ST3686 & 0119:H19 & $1 \mathrm{a}$ & - & + & + & + & + & - & - & - \\
\hline & & $12 \mathrm{ST} 455$ & EMSX11011 & ST162 & O82:H19 & 1a & - & + & + & + & + & - & - & - \\
\hline & & 12ST451 & EMSX11012 & ST3881 & 08:H16 & - & $2 \mathbf{b}$ & - & + & - & - & - & - & - \\
\hline & & $15 S T 442$ & EMSX11013 & ST3692 & O2:H45 & 1a & - & - & - & - & - & + & - & + \\
\hline & & $15 S T 447$ & EMSX11013 & ST3692 & O2:H45 & 1a & - & - & - & - & - & + & - & + \\
\hline & & $12 \mathrm{ST} 456$ & EMSX11013 & ST3692 & $\mathrm{O} 2: \mathrm{H} 45$ & 1a & - & - & - & - & - & + & - & + \\
\hline & & 13ST298 & EMSX11014 & ST906 & O74:H8 & - & $2 d$ & + & + & + & + & - & - & - \\
\hline & & $15 S T 440$ & EMSX11015 & ST906 & O74:H8 & - & $2 a, 2 d$ & + & + & + & + & - & - & - \\
\hline & & $12 \mathrm{ST} 458$ & EMSX11016 & ST906 & O74:H8 & $1 \mathrm{a}$ & $2 d$ & + & + & + & + & - & - & - \\
\hline
\end{tabular}

FIGURE 1 | Pulsed-field gel electrophoresis (PFGE) profiles of 22 non-0157 Shiga toxin-producing Escherichia coli (STEC) isolates from plateau pikas. The corresponding isolate names, PFGE patterns, sequence types (STs), serotypes, st $x_{1}$ and stx 2 subtypes, the profiles of ehxA, iha, saa, subA, and astA genes, and antibiotic resistances are listed on the right. Year of sampling is contained in the first two numbers of the isolate names: 12 means year 2012 , 13 means 2013, and 15 means 2015. Abbreviations for antibiotics are: S, Streptomycin; KF, Cephalothin. '+' means genes positive or antibiotic resistant; '-' means genes negative or antibiotic susceptible. 
negative for $k a t P$, tox $B$, the HPI genes and the 11 nle genes (Figure 1).

\section{Antibiotic Resistance}

Antimicrobial resistance was determined using 23 antibiotics. All 22 STEC isolates were resistant to penicillin G. Four and one isolates were resistant to cephalothin and streptomycin, respectively, giving resistance rates of 18.18 and $4.55 \%$. All isolates were susceptible to the remaining 20 antibiotics (Figure 1).

\section{Pulsed-Field Gel Electrophoresis (PFGE)}

The 22 non-O157 STEC isolates were analyzed by PFGE to investigate their genetic relationships. All isolates produced clear bands, and could be divided into 16 PFGE patterns (EMSX11001 to EMSX11016). An UPGMA dendrogram showed that the STEC isolates were genetically diverse with nodes linking isolates at less than 90\% similarity (Figure 1). Four isolates (12ST452, 12ST453, 12ST454, 12ST459) from intestinal contents collected in 2012, two isolates (15ST441, 15ST444) from intestinal contents collected in 2015, and three isolates (12ST456, 15ST442, and 15ST447) from intestinal contents collected in different years (two in 2015 and one in 2012) showed identical PFGE patterns, sequence type, serotype and virulence gene profile. The two isolates (15ST443, 15ST444) obtained from a single intestinal contents sample showed different PFGE patterns, serotypes and virulence gene profiles (Figure 1).

\section{Multi-locus Sequence Typing (MLST)}

A total of 12 different sequence types (STs) were identified among the 22 STEC isolates, including one novel sequence type, ST5741, resulting from a novel allele type, icd 592. ST1001 was the most frequent ST, represented by four isolates with identical PFGE pattern, serotype, virulence gene profile and antibiotic resistance pattern. Three STs (ST297, ST906, and ST3692) were represented by three isolates each. Among these, the ST297 isolates showed different PFGE patterns, serotypes and stx profiles; ST906 isolates demonstrated different PFGE patterns and stx profiles; while ST3692 isolates exhibited an identical PFGE pattern, serotype, virulence gene profile and antibiotic resistance pattern. ST40 was detected in two isolates with different resistance patterns, and the other seven STs were found only once. There is a good concordance between MLST and PFGE; isolates of the same ST generally showed the same or similar PFGE patterns (Figure 1).

\section{DISCUSSION}

The Qinghai-Tibetan plateau is a region where the altitude is high, the temperature is low and ultraviolet radiation is strong. Plateau pikas (O. curzoniae) play important roles in this ecosystem, such as recycling nutrients in soil, providing food to predators, and providing microhabitats by increasing plant richness, while their burrows provide nests for small birds and reptiles ( $\mathrm{Yu}$ et al., 2012). Plateau pikas have been proved to be a reservoir of some pathogens (Zhang et al., 2013; Yu et al., 2014). In the current research, we found that $5.2 \%(58 / 1116)$ of the plateau pika samples were positive for the stx genes by PCR and $1.88 \%(21 / 1116)$ by microbiological culture. The prevalence of STEC in plateau pikas is much lower than that in yaks from the same geographical region (Bai et al., 2013), where $11.68 \%(85 / 728)$ of samples yielded STEC strains. Notably, more than $60 \%$ of the stx-positive samples by PCR were negative by culture in this study. It was inferred that several factors may result in the failure to isolate STEC from the stx-positive samples, such as the low levels of STEC in the samples, the perturbation of high levels of background microflora, the presence of other bacteria carrying stx, the interference of free Stx phages (Quiros et al., 2015). Different STEC isolation methods may also contribute to the recovery of different serotype strains (Kalchayanand et al., 2013) and dual plating on lesser and more highly selective agars may maximize the recovery of target STEC strains (Gill et al., 2014). A possible reason for no isolation of STEC O157 and the top six non-O157 STEC strains might be the method used. In this study, we used CHROMagar ${ }^{\mathrm{TM}}$ ECC and MacConkey agars and didn't specifically target $\mathrm{O} 157$ or the top six serogroups. For instance, use of $\mathrm{O} 157$ or the top six serogruops specific immunomagnetic beads to capture the bacteria from enrichment broth with subsequent culture in selective media are required in further study to improve the isolation of these strains. Notably, STEC were similarly distributed in intestine content samples collected in the years 2012 and 2015, while the prevalence in feces was much lower, according with observations on swine STEC strains in our previous study (Meng et al., 2014).

The STEC isolates from plateau pikas were highly genetically diverse, revealed by serotyping, PFGE, MLST, and virulence gene profiles. Isolates from different samples collected in the same or different years (four isolates from year 2012, two isolates from 2015, and three isolates from 2012 and 2015) had an identical PFGE pattern, sequence type, serotype and virulence gene profile, implying some STEC clones may be widespread and persistent in this region. STEC isolates 15ST443 and 15ST444 obtained from the same intestinal contents sample showed different PFGE patterns, serotypes and virulence gene profiles, indicating that some plateau pikas could be colonized by more than one STEC clone.

The predominant serotypes $\mathrm{O} 8: \mathrm{H} 2$ and $\mathrm{O} 2: \mathrm{H} 45$ were also observed in yak STEC isolates, and $\mathrm{O} 2: \mathrm{H} 45$ was the most common serotype among yak isolates (Bai et al., 2013). Nine sequence types (ST40, ST297, ST737, ST906, ST1001, ST3686, ST3692, ST3883, and ST3884) were identified in STEC from both yaks (Sun et al., 2013) and plateau pikas, suggesting some STEC strains may be widely spread among animals in the same geographical region; however, the transmission route remains to be understood. Resistance to multiple antimicrobials has been shown in many non-O157 STEC isolates from humans, animals and food (Stephan and Schumacher, 2001). Wong et al. (2000) supported the theory that antibiotics play an 
important role in the progression of HUS and therefore the resistances of STEC may have relevance to clinical symptoms. In the present study, 20 of the 23 antimicrobial agents were active against all 22 plateau pika STEC isolates, the exceptions being penicillin $\mathrm{G}$, cephalothin and streptomycin. These results were quite different from those observed in healthy pigs in China, where only two of the 23 antimicrobial agents (imipenem and meropenem) were active against all the STEC isolates (Meng et al., 2014). The lower antibiotic resistance may due to a low prevalence of drug resistant bacteria in this ecosystem.

Shiga toxin (Stx) production, especially Stx1a, Stx2a, Stx2c, and Stx2d, has been implicated in causing severe disease and HUS, and STEC carrying Stx $2 b$ are mainly associated with diarrheal disease (Friedrich et al., 2002; Jelacic et al., 2003; Bielaszewska et al., 2006). Adherence to the intestinal epithelium and subsequent colonization are crucial for the STEC pathogenic process. STEC isolates that carry both stx 2 and eae genes are often associated with severe disease (Werber et al., 2003). Coombes et al. (2008) reported that non-LEE encoded effector (nle) genes are correlated with outbreak and HUS potential in humans. In the present study, different profiles, i.e., st $x_{1 \mathrm{a}}, s t x_{2 \mathrm{a}}, s t x_{2 \mathrm{~b}}$, $s t x_{2 \mathrm{~d}}, s t x_{1 \mathrm{a}}+s t x_{2 \mathrm{~d}}$, and $s t x_{2 \mathrm{a}}+s t x_{2 \mathrm{~d}}$, were present in the 22 non-O157 STEC isolates, but all were eae negative. Some of the isolates carried virulence genes ehxA (68.18\%), subA (63.64\%), astA (13.64\%), or adherence genes iha $(72.23 \%)$ and saa $(68.18 \%)$. Other novel virulence or adherence factors in these STEC isolates besides stx need to be further investigated. Multi-locus sequence typing identified 12 STs obtained in this study. The 32 STs of the HUSEC collection and 36 human STEC STs from the E. coli MLST database were considered to assess the potential risk of human infection by plateau pika STEC strains. None of the STs of plateau pika STEC were identical to any of the human STEC STs. Only one, ST40, with two isolates (15ST441 and 15ST444), was also observed in the HUSEC collection (Figure 2), but these two isolates harbored the low-virulence $s t x_{2 b}$ gene and no other virulence genes were detected. These data suggest that STEC in plateau pikas may have low potential to cause human disease.

In China, STEC O157 strains were detected in different animals and caused a major outbreak in 1999 (Xiong et al., 2012; Meng et al., 2013). Non-O157 STEC strains have been isolated from different animals and raw meats in China as reported in

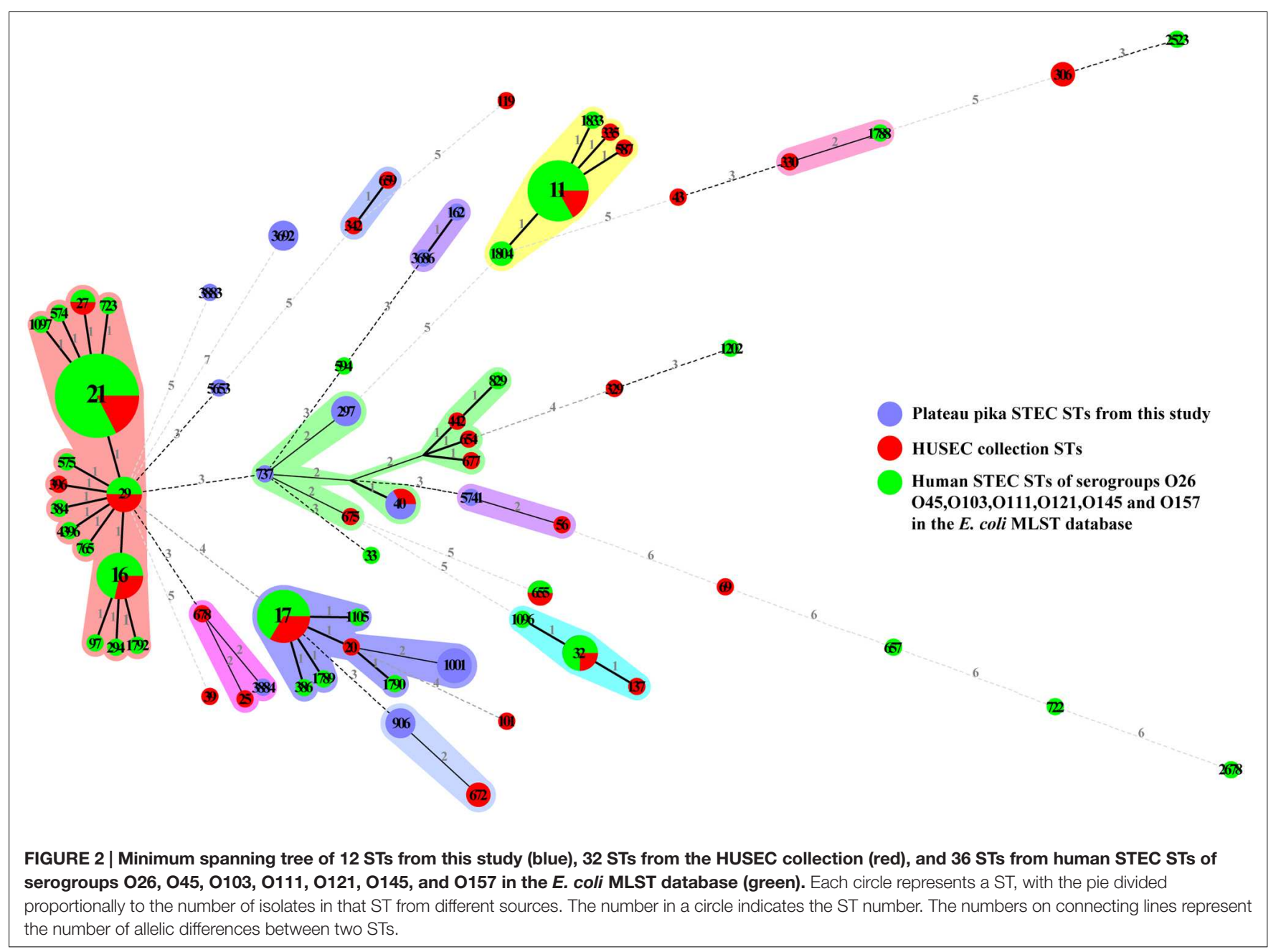


our previous studies (Bai et al., 2013, 2015; Meng et al., 2014). In recent years, STEC isolates including O157 and non-O157 serogroups have been reported in diarrheal patient by several other studies in China (Chen et al., 2014; Wang et al., 2015; Yu et al., 2015), but limited information is available regarding the phenotypic and genetic characteristics of these STEC isolates. Thus, further indepth studies on STECs from different resources in China are required to determine their pathogenic potential.

\section{CONCLUSION}

We initially revealed that plateau pikas may be a natural reservoir of STEC, extending our knowledge of the genetic diversity and reservoir host range of STEC. Based on comparison by serotypes and MLST analysis with human strains and presence of virulence genes, the majority of these pika STECs may have a low potential to cause human disease. However, further investigations are needed to assess the public health significance in Tibetans and nomadic pastoralists in this geographic region.

\section{REFERENCES}

Bai, X., Wang, H., Xin, Y., Wei, R., Tang, X., Zhao, A., et al. (2015). Prevalence and characteristics of Shiga toxin-producing Escherichia coli isolated from retail raw meats in China. Int. J. Food Microbiol. 200, 31-38. doi: 10.1016/j.ijfoodmicro.2015.01.018

Bai, X., Zhao, A., Lan, R., Xin, Y., Xie, H., Meng, Q., et al. (2013). Shiga toxin-producing Escherichia coli in yaks (Bos grunniens) from the QinghaiTibetan Plateau, China. PLoS ONE 8:e65537. doi: 10.1371/journal.pone. 0065537

Bentancor, A., Rumi, M. V., Carbonari, C., Gerhardt, E., Larzabal, M., Vilte, D. A., et al. (2012). Profile of Shiga toxin-producing Escherichia coli strains isolated from dogs and cats and genetic relationships with isolates from cattle, meat and humans. Vet. Microbiol. 156, 336-342. doi: 10.1016/j.vetmic.2011.10.030

Bielaszewska, M., Friedrich, A. W., Aldick, T., Schurk-Bulgrin, R., and Karch, H. (2006). Shiga toxin activatable by intestinal mucus in Escherichia coli isolated from humans: predictor for a severe clinical outcome. Clin. Infect. Dis. 43, 1160-1167. doi: 10.1086/508195

Brooks, J. T., Sowers, E. G., Wells, J. G., Greene, K. D., Griffin, P. M., Hoekstra, R. M., et al. (2005). Non-O157 Shiga toxin-producing Escherichia coli infections in the United States, 1983-2002. J. Infect. Dis. 192, 1422-1429. doi: $10.1086 / 466536$

Caprioli, A., Tozzi, A. E., Rizzoni, G., and Karch, H. (1997). Non-O157 Shiga toxinproducing Escherichia coli infections in Europe. Emerg. Infect. Dis. 3, 578-579. doi: 10.3201/eid0304.970425

Chen, Y., Chen, X., Zheng, S., Yu, F., Kong, H., Yang, Q., et al. (2014). Serotypes, genotypes and antimicrobial resistance patterns of human diarrhoeagenic Escherichia coli isolates circulating in southeastern China. Clin. Microbiol. Infect. 20, 52-58. doi: 10.1111/1469-0691.12188

Chui, L., Li, V., Fach, P., Delannoy, S., Malejczyk, K., Patterson-Fortin, L., et al. (2015). Molecular profiling of Escherichia coli O157:H7 and non-O157 strains isolated from humans and cattle in Alberta. Canada. J. Clin. Microbiol. 53, 986-990. doi: 10.1128/JCM.03321-14

Clinical Laboratory Standards Institute [CLSI] (2014). Performance Standards for Antimicrobial Susceptibility Testing; Twenty-Fourth Informational Supplement M100-S24. Wayne, PA: Clinical, and Laboratory Standards Institute [CLSI].

Conrad, C. C., Stanford, K., Mcallister, T. A., Thomas, J., and Reuter, T. (2014). Further development of sample preparation and detection methods for O157 and the top 6 non-O157 STEC serogroups in cattle feces. J. Microbiol. Methods 105, 22-30. doi: 10.1016/j.mimet.2014.06.020

\section{AUTHOR CONTRIBUTIONS}

$\mathrm{XB}, \mathrm{JX}, \mathrm{YX}$, and SL designed the project, analyzed data and wrote the manuscript. XT and YX collected samples. WZ, YX, HS, XL, and JP carried out the experiments.

\section{ACKNOWLEDGMENTS}

This work was supported by grants from the National Natural Science Foundation of China (81371762 and 81290345), the State Key Laboratory for Infectious Disease Prevention and Control (2015SKLID504), and the National Basic Research Priorities Program of China (2015CB554201).

\section{SUPPLEMENTARY MATERIAL}

The Supplementary Material for this article can be found online at: http://journal.frontiersin.org/article/10.3389/fmicb. 2016.00375

Cookson, A. L., Bennett, J., Thomson-Carter, F., and Attwood, G. T. (2007). Molecular subtyping and genetic analysis of the enterohemolysin gene (ehxA) from Shiga toxin-producing Escherichia coli and atypical enteropathogenic E. coli. Appl. Environ. Microbiol. 73, 6360-6369. doi: 10.1128/AEM.00316-07

Coombes, B. K., Wickham, M. E., Mascarenhas, M., Gruenheid, S., Finlay, B. B., and Karmali, M. A. (2008). Molecular analysis as an aid to assess the public health risk of non-O157 Shiga toxin-producing Escherichia coli strains. Appl. Environ. Microbiol. 74, 2153-2160. doi: 10.1128/AEM.02566-07

Doorduyn, Y., De Jager, C. M., Van Der Zwaluw, W. K., Friesema, I. H., Heuvelink, A. E., De Boer, E., et al. (2006). Shiga toxin-producing Escherichia coli (STEC) O157 outbreak, The Netherlands, September-October 2005. Euro. Surveill. 11, $182-185$.

Fields, P. I., Blom, K., Hughes, H. J., Helsel, L. O., Feng, P., and Swaminathan, B. (1997). Molecular characterization of the gene encoding $\mathrm{H}$ antigen in Escherichia coli and development of a PCR-restriction fragment length polymorphism test for identification of E. coli O157:H7 and O157:NM. J. Clin. Microbiol. 35, 1066-1070.

Frankel, G., and Phillips, A. D. (2008). Attaching effacing Escherichia coli and paradigms of Tir-triggered actin polymerization: getting off the pedestal. Cell Microbiol. 10, 549-556. doi: 10.1111/j.1462-5822.2007.01103.x

Friedrich, A. W., Bielaszewska, M., Zhang, W. L., Pulz, M., Kuczius, T., Ammon, A., et al. (2002). Escherichia coli harboring Shiga toxin 2 gene variants: frequency and association with clinical symptoms. J. Infect. Dis. 185, 74-84. doi: $10.1086 / 338115$

Friesema, I., Schimmer, B., Stenvers, O., Heuvelink, A., De Boer, E., Van Der Zwaluw, K., et al. (2007). STEC O157 outbreak in the Netherlands, SeptemberOctober 2007. Euro. Surveill. 12:E071101.1.

Gill, A., Huszczynski, G., Gauthier, M., and Blais, B. (2014). Evaluation of eight agar media for the isolation of shiga toxin-Producing Escherichia coli. J. Microbiol. Methods 96, 6-11. doi: 10.1016/j.mimet.2013.10.022

Greenland, K., De Jager, C., Heuvelink, A., Van Der Zwaluw, K., Heck, M., Notermans, D., et al. (2009). Nationwide outbreak of STEC O157 infection in the Netherlands, December 2008-January 2009: continuous risk of consuming raw beef products. Euro. Surveill 14:19129.

Gunzer, F., Bohm, H., Russmann, H., Bitzan, M., Aleksic, S., and Karch, H. (1992). Molecular detection of sorbitol-fermenting Escherichia coli $\mathrm{O} 157$ in patients with hemolytic-uremic syndrome. J. Clin. Microbiol. 30, 1807-1810.

Jelacic, J. K., Damrow, T., Chen, G. S., Jelacic, S., Bielaszewska, M., Ciol, M., et al. (2003). Shiga toxin-producing Escherichia coli in Montana: bacterial genotypes and clinical profiles. J. Infect. Dis. 188, 719-729. doi: 10.1086/376999 
Joensen, K. G., Tetzschner, A. M., Iguchi, A., Aarestrup, F. M., and Scheutz, F. (2015). Rapid and easy in silico serotyping of Escherichia coli isolates by use of whole-genome sequencing data. J. Clin. Microbiol. 53, 2410-2426. doi: 10.1128/JCM.00008-15

Kalchayanand, N., Arthur, T. M., Bosilevac, J. M., Wells, J. E., and Wheeler, T. L. (2013). Chromogenic agar medium for detection and isolation of Escherichia coli serogroups O26, O45, O103, O111, O121, and O145 from fresh beef and cattle feces. J. Food Prot. 76, 192-199. doi: 10.4315/0362-028X.JFP-12-182

Karmali, M. A., Petric, M., Lim, C., Fleming, P. C., Arbus, G. S., and Lior, H. (1985). The association between idiopathic hemolytic uremic syndrome and infection by verotoxin-producing Escherichia coli. J. Infect. Dis. 151, 775-782. doi: 10.1093/infdis/151.5.775

Mellmann, A., Bielaszewska, M., Kock, R., Friedrich, A. W., Fruth, A., Middendorf, B., et al. (2008). Analysis of collection of hemolytic uremic syndrome-associated enterohemorrhagic Escherichia coli. Emerg. Infect. Dis. 14, 1287-1290. doi: 10.3201/eid1408.071082

Meng, Q., Bai, X., Zhao, A., Lan, R., Du, H., Wang, T., et al. (2014). Characterization of Shiga toxin-producing Escherichia coli isolated from healthy pigs in China. BMC Microbiol. 14:5. doi: 10.1186/1471-2180-14-5

Meng, Q., Xiong, Y., Lan, R., Ye, C., Wang, T., Qi, T., et al. (2013). SNP genotyping of enterohemorrhagic Escherichia coli O157:H7 isolates from China and genomic identity of the 1999 Xuzhou outbreak. Infect. Genet. Evol. 16, 275-281. doi: 10.1016/j.meegid.2013.02.018

Miko, A., Pries, K., Haby, S., Steege, K., Albrecht, N., Krause, G., et al. (2009). Assessment of Shiga toxin-producing Escherichia coli isolates from wildlife meat as potential pathogens for humans. Appl. Environ. Microbiol. 75, 6462-6470. doi: 10.1128/AEM.00904-09

Oporto, B., Esteban, J. I., Aduriz, G., Juste, R. A., and Hurtado, A. (2008). Escherichia coli O157:H7 and non-O157 Shiga toxin-producing E. coli in healthy cattle, sheep and swine herds in Northern Spain. Zoonoses Public Health 55, 73-81. doi: 10.1111/j.1863-2378.2007.01080.x

Quiros, P., Martinez-Castillo, A., and Muniesa, M. (2015). Improving detection of Shiga toxin-producing Escherichia coli by molecular methods by reducing the interference of free Shiga toxin-encoding bacteriophages. Appl. Environ. Microbiol. 81, 415-421. doi: 10.1128/AEM.02941-14

Sanchez, S., Martinez, R., Rey, J., Garcia, A., Blanco, J., Blanco, M., et al. (2010). Pheno-genotypic characterisation of Escherichia coli O157:H7 isolates from domestic and wild ruminants. Vet. Microbiol. 142, 445-449. doi: 10.1016/j.vetmic.2009.10.009

Scheutz, F., Teel, L. D., Beutin, L., Pierard, D., Buvens, G., Karch, H., et al. (2012). Multicenter evaluation of a sequence-based protocol for subtyping Shiga toxins and standardizing Stx nomenclature. J. Clin. Microbiol. 50, 2951-2963. doi: 10.1128/JCM.00860-12

Shen, J., Rump, L., Ju, W., Shao, J., Zhao, S., Brown, E., et al. (2015). Virulence characterization of non-O157 Shiga toxin-producing Escherichia coli isolates from food, humans and animals. Food Microbiol. 50, 20-27. doi: 10.1016/j.fm.2015.02.007

Sigmundsdottir, G., Atladottir, A., Hardardottir, H., Gudmundsdottir, E., Geirsdottir, M., and Briem, H. (2007). STEC O157 outbreak in Iceland, September-October 2007. Euro. Surveill. 12:E071101.2.

Smith, J. L., Fratamico, P. M., and Gunther, N. W. T. (2014). Shiga toxin-producing Escherichia coli. Adv. Appl. Microbiol. 86, 145-197. doi: 10.1016/B978-0-12800262-9.00003-2

Stephan, R., and Schumacher, S. (2001). Resistance patterns of non-O157 Shiga toxin-producing Escherichia coli (STEC) strains isolated from animals, food and asymptomatic human carriers in Switzerland. Lett. Appl. Microbiol. 32, 114-117. doi: 10.1046/j.1472-765x.2001.00867.x

Sun, H., Bai, X., Zhao, A., Meng, Q., Xiong, Y., and Lu, S. (2013). Multilocus sequence typing of Shiga toxin-producing Escherichia coli isolated from yaks. Chin. J. Zoonoese 29, 1137-1142.
Tamura, K., Stecher, G., Peterson, D., Filipski, A., and Kumar, S. (2013). MEGA6: molecular evolutionary genetics analysis version 6.0. Mol. Biol. Evol. 30, 27252729. doi: 10.1093/molbev/mst197

Tozzi, A. E., Caprioli, A., Minelli, F., Gianviti, A., De Petris, L., Edefonti, A., et al. (2003). Shiga toxin-producing Escherichia coli infections associated with hemolytic uremic syndrome, Italy, 1988-2000. Emerg. Infect. Dis. 9, 106-108. doi: 10.3201/eid0901.020266

Tseng, M., Fratamico, P. M., Bagi, L., Delannoy, S., Fach, P., Manning, S. D., et al. (2014). Diverse virulence gene content of Shiga toxin-producing Escherichia coli from finishing swine. Appl. Environ. Microbiol. 80, 6395-6402. doi: 10.1128/AEM.01761-14

Wang, X., Wang, J., Sun, H., Xia, S., Duan, R., Liang, J., et al. (2015). Etiology of childhood infectious diarrhea in a developed region of china: compared to childhood diarrhea in a developing region and adult diarrhea in a developed region. PLOS ONE 10:e0142136. doi: 10.1371/journal.pone. 0142136

Wasilenko, J. L., Fratamico, P. M., Sommers, C., Demarco, D. R., Varkey, S., Rhoden, K., et al. (2014). Detection of shiga toxin-producing Escherichia coli (STEC) O157:H7, O26, O45, O103, O111, O121, and O145, and Salmonella in retail raw ground beef using the DuPont BAX(R) system. Front. Cell Infect. Microbiol. 4:81. doi: 10.3389/fcimb.2014.00081

Werber, D., Fruth, A., Buchholz, U., Prager, R., Kramer, M. H., Ammon, A., et al. (2003). Strong association between shiga toxin-producing Escherichia coli O157 and virulence genes stx 2 and eae as possible explanation for predominance of serogroup $\mathrm{O} 157$ in patients with haemolytic uraemic syndrome. Eur. J. Clin. Microbiol. Infect. Dis. 22, 726-730. doi: 10.1007/s10096-0031025-0

Wong, C. S., Jelacic, S., Habeeb, R. L., Watkins, S. L., and Tarr, P. I. (2000). The risk of the hemolytic-uremic syndrome after antibiotic treatment of Escherichia coli O157:H7 infections. N. Engl. J. Med. 342, 1930-1936. doi: 10.1056/NEJM200006293422601

Xiong, Y., Wang, P., Lan, R., Ye, C., Wang, H., Ren, J., et al. (2012). A novel Escherichia coli O157:H7 clone causing a major hemolytic uremic syndrome outbreak in China. PLoS ONE 7:e36144. doi: 10.1371/journal.pone. 0036144

Yu, F., Li, S., Kilpatrick, W. C., Mcguire, P. M., He, K., and Wei, W. (2012). Biogeographical study of plateau pikas Ochotona curzoniae (Lagomorpha, Ochotonidae). Zoolog. Sci. 29, 518-526. doi: 10.2108/zsj.29.518

Yu, J., Jing, H., Lai, S., Xu, W., Li, M., Wu, J., et al. (2015). Etiology of diarrhea among children under the age five in China: results from a five-year surveillance. J. Infect. 71, 19-27. doi: 10.1016/j.jinf.2015.03.001

Yu, Z., Cheng, K., Sun, W., Xin, Y., Cai, J., Ma, R., et al. (2014). Lowly pathogenic avian influenza (H9N2) infection in Plateau pika (Ochotona curzoniae), Qinghai Lake, China. Vet. Microbiol. 173, 132-135. doi: 10.1016/j.vetmic.2014.07.002

Zhang, X. X., Lou, Z. Z., Huang, S. Y., Zhou, D. H., Jia, W. Z., Su, C., et al. (2013). Genetic characterization of Toxoplasma gondii from Qinghai vole, Plateau pika and Tibetan ground-tit on the Qinghai-Tibet Plateau, China. Parasit. Vectors. 6:291. doi: 10.1186/1756-3305-6-291

Conflict of Interest Statement: The authors declare that the research was conducted in the absence of any commercial or financial relationships that could be construed as a potential conflict of interest.

Copyright (c) 2016 Bai, Zhang, Tang, Xin, Xu, Sun, Luo, Pu, Xu, Xiong and Lu. This is an open-access article distributed under the terms of the Creative Commons Attribution License (CC BY). The use, distribution or reproduction in other forums is permitted, provided the original author(s) or licensor are credited and that the original publication in this journal is cited, in accordance with accepted academic practice. No use, distribution or reproduction is permitted which does not comply with these terms. 